\title{
Basic Tools for Modeling Stellar and Planetary Atmospheres
}

\author{
Ivan Hubeny \\ Steward Observatory and Dept. of Astronomy, University of Arizona, Tucson, Arizona, USA \\ email: hubeny@as.arizona.edu
}

\begin{abstract}
Most popular computer codes for calculating model stellar and planetary atmospheres are briefly reviewed. A particular emphasis is devoted to our universal computer program TLuSTY (model stellar atmospheres and accretion disks), CoolTlusty (a variant of TLusTY for computing model atmospheres of substellar-mass objects such as giant planets and brown dwarfs), and SyNSPEC (an associated spectrum synthesis code). We show the highlights of actual applications of these codes which include extensive grids of fully line-blanketed non-LTE model atmospheres of $\mathrm{O}$ and $\mathrm{B}$ stars, and grids of model atmospheres of extrasolar giant planets and $\mathrm{L}$ and $\mathrm{T}$ dwarfs.
\end{abstract}

Keywords. stars: atmospheres, planets and satellites: general, radiative transfer

\section{Introduction}

The term stellar (planetary) atmosphere refers to any medium connected physically to a star (planet) from which the photons escape to the surrounding space. In other words, it is a region where the radiation, observable by a distant observer, originates. Since in the vast majority of cases the radiation is the only information about a distant astronomical object that can be obtained (exceptions being a direct detection of solar wind particles, neutrinos from the Sun and SN 1987a, or gravitational waves), all the information that is gathered about stars is derived from analysis of their radiation.

Stellar atmospheres are an example of an astrophysical medium where radiation is not only a probe of the physical state, but is in fact an important constituent. In other words, radiation in fact determines the structure of the medium, yet the medium is probed only by this radiation. This leads to a mathematical complexity of the modeling procedure, because the radiation field has to be determined self-consistently with the atmospheric structure, in order to fully extract the wealth of information encoded in an observed spectrum.

The aim of this paper is to provide a brief overview of the problems and approximations in modeling atmospheres of stars and sub-stellar mass objects - brown dwarfs and extrasolar giant planets.

\section{Model Atmospheres}

By the term model atmosphere we mean a specification of all the atmospheric state parameters as functions of position. These parameters are obtained by solving appropriate structural equations. Which equations are to be solved, and what form they attain, depends sensitively on the adopted assumptions and approximations.

\subsection{Approximations}

In order to make the overall problem tractable, one has to make a number of simplifications by invoking various approximations. The quality of an appropriate model, and 
consequently its applicability to the individual stellar types, is closely related to the degree of approximation used in the construction of the model. Needless to say, the degree of approximation critically influences the amount of computational effort to compute it. It is fair to say that the very art of computing model stellar atmospheres is to find such physical approximations that allow the model to be computed with a reasonable amount of numerical work, yet the model is sufficiently realistic to allow its use for a reliable interpretation of observed stellar data. The adopted approximations are therefore critical. There are several types of approximations that are typically made in the model construction; we shall describe the most important types in turn.

\section{Approximations of the geometry}

By the geometrical simplification we mean that either some prescribed geometrical configuration is assumed, or some special kind of overall symmetry is invoked. The goal of those simplifications is to reduce the dimensionality of the problem from a spatially 3 -dimensional problem to a $1-\mathrm{D}$ or $2-\mathrm{D}$ problem. The most popular approximations are, from simplest to more complex:

- Plane-parallel geometry, with an assumption of horizontally homogeneous layers. This decreases the number of dimensions to one: the depth in the atmosphere. This approximation is typically quite reasonable for stellar photospheres, which indeed are by several orders of magnitude thinner than the stellar radius, so the curvature effects are negligible. In the presence of horizontal inhomogeneities (such as stellar spots, accretion belts, etc.), 1-D models still have their value since in many cases one may construct different 1-D models for the individual "patches" on the surface.

- Spherical symmetry. Again, the problem is one-dimensional. The approach is used for extended atmospheres, for which the atmospheric thickness in no longer negligible with respect to the stellar radius. These models are appropriate for giants and supergiants.

- Multi-dimensional geometry. This field is enjoying a period of rapid development. We will not discuss it here (see contributions by Bonifacio, this volume; Allard, this volume).

\section{Approximations of the dynamical state of the atmosphere}

This is basically a specification of the realism of the treatment of the macroscopic velocity fields. From the simplest to the most complex the approaches are the following:

- Static models, in which the macroscopic velocity field is set to zero. These models describe a stellar photosphere.

- Models with an a priori given velocity field. In these models the velocities are taken into account explicitly, and their influence upon other state parameters, in particular the emergent radiation, is studied in detail. The most successful computer programs for computing such models are CMFGEN (Hillier \& Miller 1998; Hillier, this volume), PHOENIX (Hauschildt et al. 1997; Allard, this volume), Munich codes (FASTwIND - Santolaya-Rey et al. 1997; WM-BASIC - Pauldrach et al. 2001), and the Kiel-Potsdam code (Hamann 1985; Koesterke et al. 2002).

- Models where the velocity field is determined self-consistently by solving the appropriate hydrodynamical equations. This problem is very complicated because the wind driving force is given by the absorption of photons in thousands to millions of metal lines so the hydrodynamical equations should be solved together with at least an approximate treatment of radiative transfer in spectral lines. Such a fully self-consistent model is yet to be constructed. 
Approximations of the opacity sources

In real stellar atmospheres, there is an enormous number of possible opacity sources. The light elements $(\mathrm{H}, \mathrm{He}, \mathrm{C}, \mathrm{N}, \mathrm{O})$ have a comparatively small number of lines per ion (say $10^{2}$ to $10^{4}$ ) because of a relatively simple atomic level structure. The number of lines generally increases with increasing atomic number, and for the iron-peak elements (Fe and $\mathrm{Ni}$ being the most important ones), we have on the order of $10^{6}$ to $10^{7}$ spectral lines per ion. Models that deal with such a large number of lines are called metal line-blanketed models. There are two basic approaches to compute them, using i) Opacity Distribution Functions (ODF), routinely used in LTE, and generalized to NLTE by Anderson (1989) and Hubeny \& Lanz 1995; or ii) Opacity Sampling (OS), which is a simple Monte Carlolike sampling of frequency points (Anderson 1989; Dreizler \& Werner 1993). In fact, an "exact" method is essentially a variant of the OS with a sufficiently high resolution. An explicit comparison between results using the ODF and the OS approaches, and with various frequency resolutions in the latter, is presented e.g. in Lanz \& Hubeny (2003).

\section{Approximations concerning the thermodynamic equilibria}

Here, the issue is whether the approximation of LTE is adopted or not. In practice, LTE models may be useful only for stellar photospheres, because for extended atmospheres and/or stellar winds this approximation breaks down completely and its application would yield erroneous and misleading results.

The models that take some kind of departure from LTE into account are called nonLTE (or NLTE) models. This term is rather ambiguous because it is not a priori clear what is actually allowed to depart from LTE in a given model. In early models, the populations of only a few low-lying energy levels of the most abundant species, like $\mathrm{H}$ and He, were allowed to depart from LTE; the rest were treated in LTE. During the development of the field, progressively more and more levels were allowed to depart from LTE.

A commonly accepted rule of thumb is that for solar-type and cooler atmospheres, LTE provides an acceptable approximation, while for hotter stars, and particularly for, A, B, O stars, NLTE effects are important. Such a division is, however, misleading and even dangerous, because in essentially any kind of atmosphere there are geometric and spectral regions (outer layers; cores of strong lines), where the NLTE effects are important or even crucial.

\section{Available modeling codes}

As follows from the above, computing atmospheric structure is rather complicated. However, from the point of view of spectroscopic diagnostics of observed objects, the most important quantity is not so much a detailed structure, but rather a predicted emergent radiation. Therefore, a computed structure may be, to some extent, approximated, while the emergent radiation needs to be known as accurately as possible.

This suggests a two-step strategy. First, one computes an atmospheric structure, which may (and, in fact, has to) be computed using various approximations. Second, taking the computed structure, one calculates the emergent radiation in detail. (For instance, for normal main-sequence stars - not chemically peculiar stars - $\mathrm{Cr}$ and $\mathrm{Mn}$ do not have to be taken into account in detail or can be disregarded when computing a global atmospheric structure, while they can be taken into account when computing the emergent spectrum.) The codes that perform the first step are called the model atmosphere codes, and those performing the second step are called spectrum synthesis codes. We will briefly describe them in turn. 


\subsection{Model atmosphere codes}

There are several publicly available codes for computing model stellar atmospheres. The following list is by no means exhaustive.

For LTE models, the most popular code is ATLAS (Kurucz 1970; 1993), and MARCS (Gustafsson et al. 1975). It should be noted that most of the NLTE codes listed below can be used to calculate LTE models as well. Appropriately modified versions of PHOENIX and TLUSTY were actually used for generating grids of LTE models - see Sect. 4.1.

In the context of NLTE static models, the first publicly available NLTE model atmosphere code was the "NCAR code" (Mihalas, Heasley, \& Auer, 1975). More recently, popular and widely used codes are TMAP - Tuebingen Model Atmosphere Package (Werner 1986, 1989; Dreizler \& Werner 1993; Werner et al. 2003), and TLuSTY (Hubeny 1988; Hubeny \& Lanz 1992, 1995; Hubeny, Hummer, \& Lanz 1994). Static models are also being constructed by codes originally designed for expanding atmospheres (by setting the expansion velocity to a very low value), such as CMFGEN (Hillier \& Miller 1998) or PHOENIX (Hauschildt, Baron, \& Allard 1997; Hauschildt et al. 1999a).

\subsection{Tlusty}

The model atmosphere computer program TLUSTY has been described in several papers: Hubeny (1988) - the original version based on Complete Linearization (CL; originally developed by Auer \& Mihalas 1969); Hubeny \& Lanz (1992) - implementation of $\mathrm{Ng}$ and Kantorovich accelerations; Hubeny, Hummer \& Lanz (1994) - treatment of level dissolution and occupation probabilities; Hubeny \& Lanz (1995) - hybrid completelinearization/accelerated Lambda Iteration (CL/ALI) method, concept of superlevels and superlines; and Lanz \& Hubeny (2003) - opacity sampling method.

The program solves the basic equations (radiative transfer, hydrostatic equilibrium, radiative equilibrium, statistical equilibrium, charge and particle conservation). The previously separate variant called TLUSDISK was combined into one universal TLUSTY, which thus allows one to compute either a model stellar atmosphere, or the vertical structure of a given annulus in an accretion disk. Accretion disk models are described in detail by Hubeny \& Hubeny (1998), and Hubeny et al. (2001). Recent upgrades contain an improved treatment of convection (with several variants of the mixing-length formalism); external irradiation; Compton scattering (described in Hubeny et al. 2001); dielectronic recombination; and X-ray opacities, including the inner-shell (Auger) ionization (described in Hubeny et al. 2001). The program is fully data-oriented as far as the choice of atomic species, ions, energy levels, transitions, and opacity sources is concerned, with no default opacities built in. Both options, ODF and Opacity Sampling (OS), are offered for a treatment of metal line blanketing, but with increasing computer power the Opacity Sampling option becomes largely preferable.

Recently, we have developed a variant of TLusty called CoOLTLusty (described briefly in Hubeny, Burrows, \& Sudarsky 2003; Burrows, Sudarsky, \& Hubeny 2006) designed to compute model atmospheres of sub-stellar mass objects - brown dwarfs and giant planets. The code uses pre-calculated opacity and state-equation tables that include effects of extensive molecular opacities. Other significant upgrades with respect to TLUSTY are including the cloud formation and associated cloud opacity and scattering, and departures from chemical equilibrium (Hubeny \& Burrows 2007).

\subsection{Spectrum synthesis codes}

The spectrum synthesis codes take a previously computed or stored atmospheric structure and solve, frequency-by-frequency, the radiative transfer equation, with a sufficiently high resolution in the frequency space to provide a reliable predicted spectrum to be compared 
with observations. From the physical point of view, such a problem is much simpler than the model atmosphere construction. From the computational point of view, the only problem is connected to a necessity of dealing with extended line lists containing on the order of $10^{7}-10^{8}$ spectral line data; in the case of cool atmospheres where molecules are present, the line lists are even more extended (e.g. a line list for water lines contain over $10^{9}$ lines).

Here, we only briefly mention two codes. The first is Kurucz's SYNTHE, specfically designed to provide detailed emergent spectra for Kurucz model atmospheres.

A spectrum synthesis code for providing detailed synthetic spectra for TLUSTY models is called SYNSPEC. The code is also able to provide synthetic spectra for Kurucz and TMAP models, and with a slight modification of input routines, for any other models. The original reference is Hubeny, Štefl, \& Harmanec (1985); these days it is available on the web either at the TLUSTY website, http://nova.astro.umd.edu, or, its latest versions, on http://aegis.as.arizona.edu/ hubeny/pub/synspec49.tar.gz.

There is also a useful, IDL-based graphical package called SYNPLOT, which is a wrapper around SYNSPEC to enable an easy calculation of synthetic spectra based on setting appropriate keywords without the necessity of preparing the corresponding input files. It also provides a graphical user interface for a synthetic spectrum and its fitting to the observed spectrum. When used in conjunction with the OSTAR2002 and BSTAR2006 grids (see below), SYNPLOT allows for an easy evaluation of synthetic spectra for any values of basic stellar parameters, not just the gridpoint values. The program is available on http://aegis.as.arizona.edu/ hubeny/pub/synplot.tar.gz.

\section{Existing model atmosphere grids}

\subsection{LTE models}

The most extensive grid of LTE plane-parallel line-blanketed models is that of Kurucz $(1979 ; 1993)$, which is widely used by the astronomical community. The grid covers effective temperatures between $3500 \mathrm{~K}$ and $50,000 \mathrm{~K}, \log g$ between -1 and 5 , and for several metallicities.

Using the MARCS code, Gustafsson et al. (1975) generated their original grid of models for cool stars, with $T_{\text {eff }}$ between 3750 and $6000 \mathrm{~K}, \log g$ between 0.75 and 3.0, and metallicities $-3.0 \leqslant[M / H] \leqslant 0$. Recently, Gustafsson et al. (2008) made public a new, very extensive grid of MARCS model atmospheres, with $T_{\text {eff }}$ between 2500 and $8000 \mathrm{~K}$, $\log g$ between -1 and 5 , and metallicities $-5 \leqslant[M / H] \leqslant 1$. They also include "CNcycled" models with $\mathrm{C} / \mathrm{N}=4.07$ (solar), 1.5, and 0.5 ; and $\mathrm{C} / \mathrm{O}$ from 0.09 to 5 , which represents stars of spectral types $\mathrm{R}, \mathrm{S}$, and $\mathrm{N}$.

Hauschildt et al. (1999a, b) used their code PHOENIX to generate a grid of LTE spherical models for cool stars, called NextGen, with $T_{\text {eff }}$ between 3000 and $10000 \mathrm{~K}$, with step $200 \mathrm{~K}$; $\log g$ between 3.5 and 5.5, with step 0.5 ; and metallicities $-4.0 \leqslant$ $[M / H] \leqslant 0$. Another grid (Allard et al. 2000) is for pre-main-sequence cool stars with $T_{\text {eff }}$ between 2000 and $6800 \mathrm{~K}, \log g$ between 2 and 3.5, with step 0.5; stellar mass $M=0.1 M_{\odot}$; and metallicities $-4.0 \leqslant[M / H] \leqslant 0$. The models are available on-line at www.hs.uni-hamburg.de/EN/For/ThA/phoenix/index.html. A detailed comparison between the ATLAS and NextGen models was performed by Bertone et al. (2004).

\subsection{NLTE models}

There are several partial grids of NLTE models for various stellar types, mostly of hot stars. The models constructed by the TMAP code for very hot white dwarfs, subdwarfs, and pre-white dwarfs (also known as the PG 1159 stars) are available on-line at http://astro-uni-tuebingen.de/r̃auch/TMAP/TMAP.html. Rauch \& Werner (2009) 
describe the so-called Virtual Observatory, which is a web-based interface that enables a user either to extract already computed models, or generate specific models using TMAP, for very hot objects (hottest white dwarfs; super-soft X-ray sources).

Our effort culminated in the construction of a grid of NLTE fully-blanketed model atmospheres for O stars (OSTAR2002; Lanz \& Hubeny 2003) and early B stars (BSTAR2006; Lanz \& Hubeny 2007). We believe that these grids, which each took several years of computer time on several top-level workstations, represent a more or less definitive grids of models in the context of 1-D plane-parallel geometry, with hydrostatic and radiative equilibrium, and without any unnecessary numerical approximations.

The basic characteristics are as follows: The OSTAR2002 grid contains 680 individual model atmospheres for 12 values of $T_{\text {eff }}$ between 27,500 and $55,000 \mathrm{~K}$, with a step of 2,500 $\mathrm{K}$, and 8 values of $\log g$, and for 10 metallicities: 2, 1, 1/2, 1/5, 1/10, 1/30, 1/50, 1/100, $1 / 1000$, and 0 times the solar metal composition. The following species are treated in NLTE: H, He, C, N, O, Ne, Si, P, S, Fe, Ni, in all important stages of ionization; which means that there are altogether over 1000 (super)levels to be treated in NLTE, about $10^{7}$ lines, and about 250,000 frequency points to describe the spectrum.

The BSTAR2006 grid is similar. It contains 1540 individual models for 16 values of $T_{\text {eff }}$ between 15,000 and $30,000 \mathrm{~K}$, with a step of $1000 \mathrm{~K}$, and for 6 metallicities: 2 , $1,1 / 2,1 / 5,1 / 10$, and 0 times solar. The species treated in NLTE are the same as in OSTAR2002, adding $\mathrm{Mg}$ and $\mathrm{Al}$, but removing Ni. There are altogether about 1450
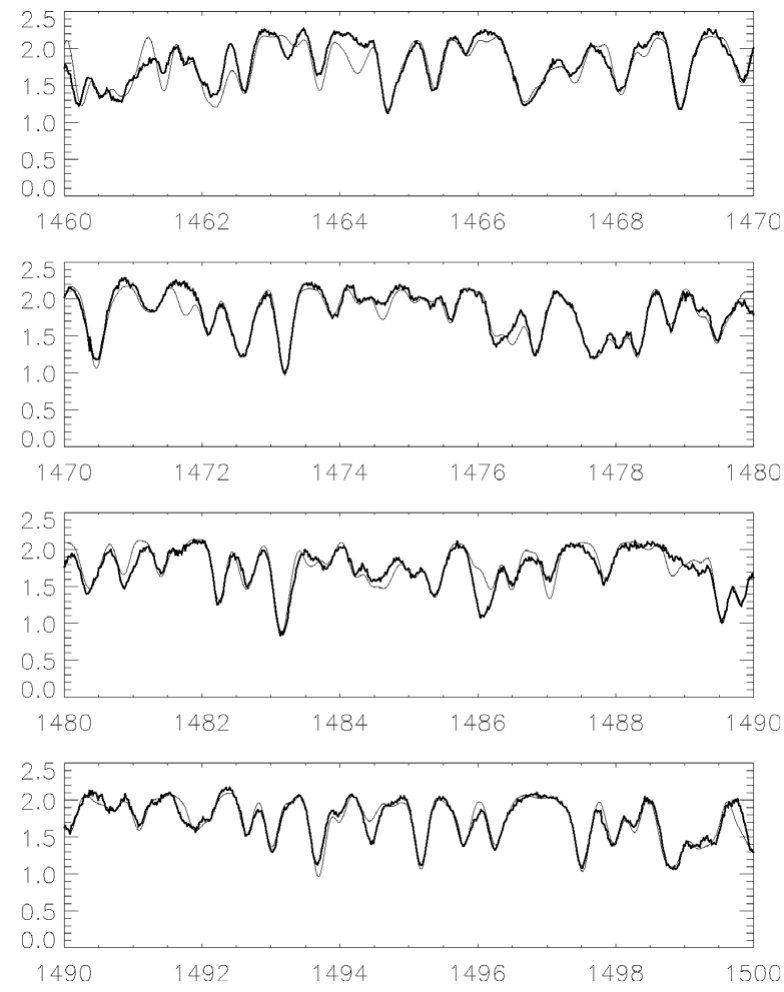

Figure 1. A comparison of the observed HST/GHRS flux for 10 Lac (heavy line) and the predicted flux from the fully blanketed NLTE model atmosphere with $T_{\text {eff }}=33,500 \mathrm{~K}, \log g=3.85$, and for the solar abundances of all species (thin line). The abscissa is the wavelength in $\AA$, and the ordinate is the flux in $10^{-9} \mathrm{erg} \mathrm{cm}^{-2} \mathrm{~s}^{-1} \AA^{-1}$. Most spectral features are lines of Fe IV, Fe $\mathrm{V}$, Ni IV, and Ni V. A difference between theory and predictions is hardly seen on the plots. 
(super)levels treated in NLTE, about $10^{7}$ lines, and about 400,000 frequency points. The models for both grids are available on-line at http://nova. astro.umd. edu.

As an example, we present in Fig. 1 a sample of the predicted flux for a model for $T_{\text {eff }}=33,500 \mathrm{~K}, \log g=3.85$, and a high-resolution, high signal-to-noise observation of a late-O main-sequence star 10 Lac secured by the Goddard High Resolution Spectrograph (GHRS) aboard the Hubble Space Telescope (Lanz et al., in prep). The agreement between observations and predictions is excellent, and demonstrates the power of the present-day model atmospheres of early-type stars.

\section{Atmospheres of Substellar-Mass Objects}

The two most interesting and important classes of the substellar-mass objects (SMO) are $\mathrm{L}$ and $\mathrm{T}$ dwarfs (sometimes referred to as brown dwarfs), and extrasolar giant planets (EGP). We stress that from the point of view of atmospheric modeling, these two types of objects are identical.

Comparing the effort needed to model the atmospheres of SMOs and classical stars, there are advantages and disadvantages. On the plus side, the atmosphere of the substellar mass objects can be treated in LTE (because these are rather cool and dense), and the radiation pressure is negligible. On the minus side, there are several serious complications: i) Molecular opacities. ii) Condensation of certain compounds and a formation of clouds. This involves several related problems, such as the distribution of cloud particle sizes, the position and extent of the cloud deck, cloud opacity and scattering cross-sections, and a strong anisotropy of cloud (Mie) scattering. iii) Complicated state equation that needs to solve a complex chemical network, even in a relatively simple case of chemical equilibrium. iv) There may be departures from chemical equilibrium, both because of photochemistry, and because of advection of certain species resulting from a long chemical reaction time compared to an atmospheric mixing time (e.g., Hubeny \& Burrows 2007); and v) strong irradiation, particularly in the case of close-in EGPs.

There are several computer programs that are being currently used to produce models of SMO atmospheres; the following three are used most actively: i) a code used by Fortney and coworkers (e.g., Fortney et al. 2005); ii) the PHOEnIX-based code (e.g., Barman et al. 2001); and our code CoolTlusty, which is a variant of Tlusty. It is described briefly in Hubeny, Burrows, \& Sudarsky 2003; and Sudarsky, Burrow, \& Hubeny 2003. It solves equations of radiative+convective equilibrium, state equation, (hydrostatic equilibrium is satisfied automatically), self-consistently with the radiative transfer equation. An upgrade to treat departures from chemical equilibrium is described in Hubeny \& Burrows (2007). The code has been used to generate extensive grids of EGP model atmospheres (Sudarsky, Burrows, \& Hubeny 2003), L and T dwarfs both with chemical equilibrium (Burrows, Sudarsky \& Hubeny 2006), and with departures from equilibrium (Hubeny \& Burrows 2007).

\section{References}

Allard, F. Hauschildt, P. H., \& Schweitzer 2000, ApJ, 539, 366

Anderson, L. S. 1989, ApJ, 339, 588

Auer, L. H. \& Mihalas, D. 1969, ApJ, 158, 641

Barman, T. S., Hauschildt, P. H., \& Allard, F. 2001, ApJ, 556, 885

Bertone, E., Buzzoni, A., Chavez, M., \& Rodriguez-Merino, L. H. 2004, AJ, 128, 829

Burrows, A., Sudarsky, D., \& Hubeny, I. 2006, ApJ, 640, 1063

Dreizler, S. \& Werner, K. 1993, A\& A, 278, 199 
Gustafsson, R., Bell, R. A., Eriksson, K., \& Nordlund, Å. 1975, A\&A, 42, 407

Gustafsson, R., Edvardsson, B., Eriksson, K., Jørgensen, U. G., Nordlund, Å., \& Plez, B. 2008, $A \mathscr{S} A, 486,951$

Fortney, J. J., Marley, M. S., Lodders, K., Saumon, D., \& Freedman, R. 2005, ApJ (Letters), 627, L69

Hamann, W.-R. 1985, A\&A, 148, 364

Hauschildt, P. H., Baron, E., \& Allard, F. 1997, ApJ, 483, 390

Hauschildt, P. H., Allard, F., \& Baron, E. 1999a, ApJ, 512, 377

Hauschildt, P. H., Allard, F., Ferguson, J., \& Baron, E. 1999b, ApJ, 525, 871

Hillier, D. J. \& Miller, D. L. 1998, ApJ, 496, 407

Hubeny, I. 1988, Comp. Phys. Commun., 52, 103

Hubeny, I., Blaes, O., Krolik, J. H., \& Agol, E. 2001, ApJ, 559, 680

Hubeny, I. \& Burrows, A. 2007, ApJ, 669, 1248

Hubeny, I. Burrows, A., \& Sudarsky, D. 2003 ApJ, 594, 1011

Hubeny, I. \& Hubeny, V. 1998, ApJ, 505, 558

Hubeny, I., Hummer, D. G., \& Lanz, T. 1994, A\&A, 282, 151

Hubeny, I. \& Lanz, T. 1992, A\&A, 262, 501

Hubeny, I. \& Lanz, T. 1995, ApJ, 439, 875

Hubeny, I., Štefl, S., \& Harmanec, P. 1985, Bull. Astron. Inst. Czechosl., 36, 214

Koesterke, L., Hamann, W.-R., \& Graefener, G. 2002, A\&A, 384, 562

Kurucz, R. L. 1970, Smithsonian Astrophys. Obs. Spec. Rep. No. 309

Kurucz, R. L. 1979, ApJS, 40, 1

Kurucz, R. L. 1993, ATLAS9 Stellar Atmosphere Programs and $2 \mathrm{~km} / \mathrm{s}$ Grid, Kurucz CD-ROM 13 (Cambridge, Mass: SAO)

Lanz, T. \& Hubeny, I. 2003, ApJS, 146, 417

Lanz, T. \& Hubeny, I. 2007, ApJS, 169, 83

Mihalas, D. 1978, Stellar Atmospheres, (2nd ed., Freeman, San Francisco)

Mihalas D., Heasley J. N., \& Auer L. H. 1975, A Non-LTE model stellar atmospheres computer program, NCAR-TN/STR-104, (NCAR, Boulder)

Pauldrach, A. W. A., Hoffmann, T. L., \& Lennon, M. 2001, AESA, 375, 161

Rauch, T. \& Werner, K. 2009, in: I. Hubeny et al., (eds.) Recent Directions in Astrophysical Quantitative Spectroscopy and Radiation Hydrodynamics, AIP Conf. Proc, 1171, p. 85

Santolaya-Rey, A. E., Puls, J., \& Herrero, A. 1997, A\& $A, 323,488$

Sharp, C. S. \& Burrows, A. 2007, ApJS, 168, 140

Sudarsky, D., Burrows, A., \& Hubeny, I. 2003, ApJ, 588, 1121

Werner, K. 1986, A\& A, 161, 177

Werner, K., Deetjen, J. L., Dreizler, S., Nagel, T., Rauch, T., \& Schuh, S. L. 2003, in: I. Hubeny, D. Mihalas, \& K. Werner (eds.), Stellar Atmosphere Modeling, ASP Conf. Ser. vol. 288, (ASP, San Francisco 2003), p. 31

\section{Discussion}

K. Allers: Are the model grids (particularly the CoolTlusty models) available online?

I. Hubeny: Yes, they are available on Adam Burrows' website at http://www . astro.princeton.edu/ burrows/. 